University of Nebraska - Lincoln

DigitalCommons@University of Nebraska - Lincoln

Lawrence G. Harshman Publications

Papers in the Biological Sciences

2005

Insulin Signaling Is Necessary for Vitellogenesis in Drosophila melanogaster Independent of the Roles of Juvenile Hormone and Ecdysteroids: Female Sterility of the chicol Insulin Signaling Mutation Is Autonomous to the Ovary

\author{
David S. Richard \\ Susquehanna University \\ Robert Rybczynski \\ University of North Carolina \\ Thomas G. Wilson \\ Colorado State University \\ Yue Wang \\ University of Nebraska-Lincoln \\ Marta L. Wayne \\ University of Florida, Gainesville, FL
}

Richard, David S.; Rybczynski, Robert; Wilson, Thomas G.; Wang, Yue; Wayne, Marta L.; Zhou, You; Partridge, Linda; and Harshman, Lawrence G., "Insulin Signaling Is Necessary for Vitellogenesis in Drosophila melanogaster Independent of the Roles of Juvenile Hormone and Ecdysteroids: Female Sterility of the chico1 Insulin Signaling Mutation Is Autonomous to the Ovary" (2005). Lawrence G. Harshman Publications. 15.

http://digitalcommons.unl.edu/biosciharshman/15

This Article is brought to you for free and open access by the Papers in the Biological Sciences at DigitalCommons@University of Nebraska - Lincoln. It has been accepted for inclusion in Lawrence G. Harshman Publications by an authorized administrator of DigitalCommons@University of Nebraska - Lincoln. 
See next page for additional authors

Follow this and additional works at: http://digitalcommons.unl.edu/biosciharshman 


\section{Authors}

David S. Richard, Robert Rybczynski, Thomas G. Wilson, Yue Wang, Marta L. Wayne, You Zhou, Linda Partridge, and Lawrence G. Harshman 
Published in the Journal of Insect Physiology 51 (2005), pp. 455-464; doi:10.1016/j.jinsphys.2004.12.013 Copyright (C) 2005 Elsevier Ltd. Used by permission.

Submitted August 13, 2004; revised December 21, 2004; accepted December 22, 2004.

\title{
Insulin Signaling Is Necessary for Vitellogenesis in Drosophila melanogaster Independent of the Roles of Juvenile Hormone and Ecdysteroids: Female Sterility of the chico $^{1}$ Insulin Signaling Mutation Is Autonomous to the Ovary
}

\author{
David S. Richard ${ }^{1}$, Robert Rybczynski², Thomas G. Wilson ${ }^{3}$, Yue Wang ${ }^{4}$, \\ Marta L. Wayne ${ }^{5}$, You Zhou ${ }^{6}$, Linda Partridge ${ }^{7}$, \\ and Lawrence G. Harshman ${ }^{4}$
}

1. Department of Biology, Susquehanna University, Selinsgrove, Pennsylvania 17870, USA

2. Department of Biology, University of North Carolina, Chapel Hill, North Carolina 27599, USA

3. Department of Biology, Colorado State University, Fort Collins, Colorado 80523, USA

4. School of Biological Sciences, University of Nebraska-Lincoln, 348 Manter Hall, Lincoln, Nebraska 68588, USA

5. Department of Zoology, University of Florida, Gainesville, Florida 32611, USA

6. Microscopy Core Facility, Beadle Biotechnology Center, University of Nebraska-Lincoln, Lincoln, Nebraska 68588, USA

7. Department of Biology, University College London, London WC1E 6BT, UK

Corresponding author - Lawrence G. Harshman, email lharsh@unlserve.unl.edu

\begin{abstract}
It has been suggested that insulin signaling mutations of Drosophila melanogaster are sterile and longlived because of juvenile hormone $(\mathrm{JH})$ and ecdysteroid deficiency. However, female sterility of an insulin/IGF-like signaling mutant $\left(\mathrm{Chico}^{1}\right)$ of D. melanogaster is not mediated by downstream systemic
\end{abstract}


signaling in terms of major alterations in $\mathrm{JH}$ or ecdysteroid levels. chico ${ }^{1}$ is a null mutation in the insulin substrate protein (CHICO) gene of D. melanogaster. Homozygous chico females are sterile and their oocytes do not mature beyond the last previtellogenic stage. Homozygous chico ${ }^{1}$ females exhibit approximately wild-type rates of $\mathrm{JH}$ biosynthesis, ovarian release of ecdysteroids and haemolymph ecdysteroid levels, suggesting that these two major hormone systems play no role in producing the sterility. Previtellogenic wild-type ovaries transplanted into homozygous chico females underwent vitellogenesis, showing that systemic factors present in mutant females are sufficient to support normal vitellogenesis. chico ${ }^{1}$ ovaries transplanted into wild-type females did not undergo vitellogenesis indicating that $\mathrm{CHICO}$ is necessary in the ovary for vitellogenic maturation. The ovary transplant experiments corroborate the endocrine results and demonstrate that insulin/insulin-like signaling (IIS) is necessary for vitellogenesis even when sufficient levels of JH, ecdysteroids or other factors are present.

Keywords: insulin receptor substrate, Chico, Drosophila, ovarian development, receptor mediated endocytosis

Abbreviations: IIS, insulin/insulin-like growth factor-like signaling; IGF, insulin-like growth factor; YP, yolk protein; CA, corpora allata; DInr, Drosophila insulin receptor; JH, juvenile hormone; JHB3, juvenile hormone bisepoxide; IRS, insulin receptor substrate; INR, insulin receptor

\section{Introduction}

The D. melanogaster chico gene encodes an insulin receptor substrate (IRS)-like protein that is similar to vertebrate IRS1-4 (Bohni et al., 1999). Homozygous chico ${ }^{1}$ mutant females are sterile and do not develop vitellogenic eggs (Bohni et al., 1999; Drummond-Barbosa and Spradling, 2001). The rate of previtellogenic oocyte production in $c h i c 0^{1}$ females shows a greatly reduced response to a protein-rich diet, indicating a role for insulin/insulin-like growth factor-like signaling (IIS) in regulating the association between reproductive rate and nutrients (Drummond-Barbosa and Spradling, 2001). Females heteroallelic for mutations in the Drosophila insulin receptor (DInr) are also sterile, and have been reported to have lowered juvenile hormone (JH) biosynthesis to approximately $25 \%$ of that of wild type (Tatar et al., 2001). Similarly, DInr mutations exhibit lowered ecdysteroid release from the ovary (Tu et al., 2002). Exposure of these mutant DInr females to the JH analogue methoprene restored vitellogenesis, leading these workers to conclude that JH was a systemic mediator of the sterility seen in these mutant DInr females. JH has been thought to play a predominant role in controlling vitellogenesis (Bownes, 1989; Kelly, 1994; Nijout, 1994), but its direct effect on yolk protein (YP) endocytosis remains uncertain (Raikhel and Dhadialla, 1992). Richard et al. (1998, 2001a, b) implicated a role for ecdysteroids instead. In the present study, we show that IIS is necessary for the onset, and presumably the maintenance, of vitellogenesis in D. melanogaster irrespective of the roles of JH and ecdysteroids. 


\section{Materials and methods}

\subsection{Genotypes, culture conditions, body weight, and statistical analysis}

The $c h i c 0^{1}$ stock used for the present study was backcrossed to flies from the outbred wildtype Dahomey population; the $C y$ balancer chromosome used to maintain the chico ${ }^{1}$ mutation in stock has been retained (Clancy et al., 2001). chico ${ }^{1}$ homozygotes were collected from this stock. Dahomey females were collected from an outbreeding population. Unless otherwise described, females heterozygous for chico $^{1}$ were obtained by crossing homozygous chico ${ }^{1}$ males to virgin Dahomey females. For all experiments, flies were reared at $241 \mathrm{C}$ under constant illumination at standardized density (75 eggs per vial) on the Drosophila medium described in Carlson and Harshman (1999) and only virgin females of each genotype were used. Ovarian maturity was determined according to King (1970). The mean fresh weight of chico $^{1}$ females $(0.60 \mathrm{mg})$ was $50 \%$ that of Dahomey females $(1.21 \mathrm{mg})$. Consequently, for normalization to body weight, measurements for $c h i c o^{1}$ females were doubled. Unless otherwise stated, an analysis of variance (ANOVA) was used for the statistics.

\subsection{Ovary transplantation}

Single immature ovaries were taken from chico $^{1}$ homozygotes and transferred into a reproductively capable female. Heterozygote and Dahomey females were the reproductively capable females used as hosts for ovaries from chico $^{1}$ homozygotes. The reciprocal experiment was to surgical implant a single immature ovary from the heterozygote, a genotype that actively produced eggs, into a chico ${ }^{1}$ homozygote female. Ovaries from newly eclosed females are relatively small and contain only previtellogenic oocytes even in reproductively capable genotypes. Single ovaries taken from $1-4 \mathrm{~h}$ post-eclosion adults were transplanted into host females of the same age. The hosts were dissected 4 days later; both host ovaries and the transplanted ovary were examined (Wilson, 1982). The criterion for vitellogenic oocyte maturation throughout the study was the presence of stage 8 , or later, vitellogenic oocytes.

\subsection{Methoprene exposure}

chico $^{1}$ females were exposed to ZR-2008 (s-methoprene), the active enantiomer of methoprene (Wilson and Fabian, 1986) which is a JH analog. Exposure was either by topical administration in $0.25-0.5 \mu \mathrm{l}$ of acetone or by continuous exposure to methoprene in vapor. For exposure by vapor, a cotton plug is permeated with the methoprene which is volatile and the plug is used to cap a vial with flies (Wilson and Chaykin, 1985, Tatar et al., 2001). For the former, doses of 20-200 ng per fly were applied at day 2-3 post-eclosion and females dissected four days later. chico ${ }^{1}$ females were continuously exposed to methoprene vapor while on fresh food. The efficacy of the methoprene used in these experiments was regularly verified using reproductively capable females in which it stimulated vitellogenesis and egg production.

\subsection{JH biosynthesis}

The radiochemical assay for $\mathrm{JH}$ production in vitro, based on that developed by Tobe and Pratt (1974), was carried out in minimal essential medium (MEM) supplemented with L- 
[ ${ }^{3} \mathrm{H}$-methyl]-methionine (Amersham, specific activity $81 \mathrm{Ci} / \mathrm{mmol}$ ) as the methyl-group donor, and with the JH-esterase inhibitor octyl-1, 1,1-trifluoropropanone (OTFP) to a concentration of $0.5 \mu \mathrm{M}$ as described previously (Richard et al., 1989; Altaratz et al., 1991). Single adult female CA or isolated 3rd instar larval brain-ventral ganglion-ring gland complexes (as positive controls) were incubated for $2 \mathrm{~h}$ at $25^{\circ} \mathrm{C}$ in the presence or absence of 20 $\mu \mathrm{M}$ farnesoic acid (FA a JH precursor kindly provided by Dr. Stephen S. Tobe, University of Toronto). Scintillation counts of hexane extracts of the incubation medium were used to assess JH biosynthesis by each CA (each data point). Pooled hexane fractions were subjected to silica gel thin layer chromatography with a JH III standard visualized under UV light as described previously (Richard et al., 1989).

\subsection{Ecdysteroid assays}

Ecdysteroids were quantified by radioimmunoassay (RIA), using the SHO3 antibody (Kiriishi et al., 1990), as described by Warren et al. (1984) with modifications. Two ovaries from each female were incubated in $10 \mu \mathrm{l}$ of Grace's medium at $25^{\circ} \mathrm{C}$ for $5 \mathrm{~h}$. Each replicate determination of ecdysteroids released from ovaries was based on 40-60 separately incubated pairs of ovaries. At $24 \mathrm{~h}$ post-eclosion, two replicate determinations were made for the homozygous chico ${ }^{1}$ mutant, the $c h i c 0^{1} /$ Dahomey heterozygote and Dahomey; at $96 \mathrm{~h}$ seven replicate determinations were made for the mutant, two for the heterozygote and sixteen for Dahomey. Ecdysteroids released into incubation solution were extracted in $70 \%$ methanol for 4 days at $4{ }^{\circ} \mathrm{C}$. The extraction time and temperature were designed to match those in a previous study (Harshman et al., 1999).

Haemolymph ecdysteroids were quantified as described in Harshman et al. (1999) except that cells were removed from the haemolymph samples by centrifugation at 13,000 $\times$ $g$ for $10 \mathrm{~min}$ before extraction of ecdysteroids from the supernatant. For each replicate determination, haemolymph was drawn from 150-200 females of each genotype. There were two replicate determinations of haemolymph ecdysteroids per genotype on day 1 posteclosion and three replicate determinations for each genotype on day 4 post-eclosion.

\subsection{Ovariole number and nurse cell counts}

Ovariole numbers were determined as described by Coyne et al. (1991) by counting the ovarioles from both ovaries from 20 females per genotype. Nurse cells were counted in stage 7 egg chambers from 10-15 females per genotype (1-4 chambers/female). The number of nurse cells was determined by three independent counts of each egg chamber using a confocal microscope and an optical Z-series of Systox-green-stained ovaries.

\subsection{Yolk protein receptor $m R N A$ abundance measurement by semi-quantitative RT-PCR}

For reverse transcriptase (RT) PCR of the YP-receptor gene (yolkless), the following primers were used to amplify a predicted 409 bp fragment: 5'-CAACATCCAGAACGAAGCGG-3' forward primer; 5'-CAACTTGGCATCCACCCAGTAG-3' backward primer. The PCR reaction was conducted with $1 \mu \mathrm{g}$ total RNA using a Stratagene ProStar RT-PCR kit $\left(52^{\circ} \mathrm{C}\right.$ annealing temperature, denaturation at $95^{\circ} \mathrm{C}$, extension at $68^{\circ} \mathrm{C}$ ). RNA was extracted from ovaries using the Qiagen RNeasy kit. 


\subsection{Western blots for yolk protein}

Western blots for YP (1 $\mu$ g protein per lane) were conducted as described by Richard et al. (2001a) using a YP antibody kindly provided by Mary Bownes (University of Edinburgh).

\section{Results}

\subsection{Ovary transplants}

We tested the importance of systemic factors in the sterility phenotype of chico $^{1}$ homozygote females by reciprocal ovary transplantations (table 1). The characteristics of all egg stages is well established (King, 1970); stages 1-7 are previtellogenic and 8-14 are vitellogenic. The stages are easily visualized and scored under a dissecting microscope at 200X. In particular, previtellogenic stage 7 oocytes and vitellogenic stage 8 oocytes are readily and unequivocally distinguishable. In the case of reproductively capable ovary transplants into chico $^{1}$, a robust vitellogenic response was seen in five implants, including 15-20 vitellogenic oocytes spanning the entire range of stage 8 to mature stage 14 eggs in each ovary. In every instance, the oocytes of host $c h i c o^{1}$ ovaries terminated development at previtellogenic stage-7, typical of the mutant phenotype. In the reciprocal experiment, transplanted chico ${ }^{1}$ ovaries failed to develop oocytes beyond stage 7 even though the chico/Dahomey ( $N$ $=7$ ) or Dahomey $(N=2)$ host ovaries became fully vitellogenic in every recipient female. Therefore, chico ${ }^{1}$ ovaries failed to develop vitellogenic oocytes even in a host environment that supports normal levels of vitellogenesis and egg production, and chico ${ }^{1}$ females provided systemic factors necessary for vitellogenic oocyte maturation.

Table 1. Vitellogenic oocyte development following ovary transplantation between chico $^{1}$ homozygotes and chico $^{1} /$ Dahomey heterozygote females

\begin{tabular}{lllcc} 
& & & \multicolumn{2}{c}{ Implant ovaries } \\
\cline { 4 - 5 } Host & Implant & Post-implant hosts & Vitellogenic & Non-vitellogenic \\
\hline chico $^{1 / D a h o m e y ~}$ & chico $^{1}$ & Ovaries vitellogenic & 7 & 0 \\
Dahomey & chico $^{1}$ & Ovaries vitellogenic & 0 & 2 \\
chico $^{1}$ & chico $^{1 / D a h o m e y ~}$ & No vitellogenesis & 5 & 1 \\
chico $^{1 / D a h o m e y}$ & chico $^{1 / D a h o m e y ~}$ & Ovaries vitellogenic & 9 & 0 \\
\hline
\end{tabular}

\subsection{Methoprene administration}

The failure of chico $^{1}$ ovaries to undergo vitellogenesis when transplanted into wild-type hosts suggested that exogenous $\mathrm{JH}$ analog administration would fail to stimulate vitellogenesis of $c h i c 0^{1}$ females. We tested this hypothesis by exposure of $c h i c 0^{1}$ to methoprene. The two modes of exposure were topical administration using a range of methoprene concentrations (20-200 ng per fly) and by exposure to methoprene vapor. Topical application of methoprene, including the highest dose, which is considerably more than required for a biological response, did not stimulate vitellogenesis or egg production in chico $^{1}$ homozygotes at 1 week after application $(N=15)$. Methoprene vapor also failed to initiate vitellogenesis or egg production in newly eclosed chico $^{1}$ females exposed for 4 days beginning at 
1-6 h post-eclosion $(N=17)$, or older females after 6 days of exposure beginning at 3-4 days post-eclosion $(N=23)$.

\subsection{JH biosynthesis}

Previous research reported that insulin receptor mutation synthesized relatively low levels of JHs (Tatar et al., 2001) which share a range of phenotypes with chico including sterility. We measured the in vitro rate of production of JH. For all genotypes at both ages posteclosion, almost all of the $\mathrm{JH}$ produced in vitro was $\mathrm{JHB}_{3}$. Specifically, the maximum amount of JHIII produced from a single corpora allata in any assay was less than $5 \%$ and the remainder was $\mathrm{JHB}_{3}$. In the first experiment (fig. 1A), we assayed homozygous chico ${ }^{1}$ at 24 and $96 \mathrm{~h}$, a heterozygote genotype consisting of $c h i c 0^{1}$ over a balancer chromosome at $24 \mathrm{~h}$, and Dahomey females at 24 and $96 \mathrm{~h}$. The analysis of variance on the raw data did not indicate an effect of age $(P=0.6219)$, genotype $(P=0.7627)$, nor interaction between age and genotype $(P=0.3074)$. However, when JH synthesis was normalized for body size (fig. $3 A)$, there was an effect of genotype $(P<0.0001)$, but not of age $(P=0.2503)$ nor of interaction between age and genotype $(P=0.2424)$. Per unit weight, chico ${ }^{1}$ females synthesized JH at almost two times the rate of the other genotypes. There was no significant difference between chico $^{1}$ homozygotes and wildtype at 24 or $96 \mathrm{~h}$ posteclosion $(P<0.2500)$. However, the normalized data indicated greater JH biosynthesis by chico $0^{1}$ females at $24 \mathrm{~h}(P=0.0006)$ and $96 \mathrm{~h}(P=0.0162)$ post-eclosion. JH biosynthesis by heterozygote females at 24 and 96 $\mathrm{h}$ was compared with that by Dahomey females at the same ages, using a second batch of labeled precursor (fig. 1B). Statistical analysis of the data indicated that there was a significant interaction between genotype and age for the heterozygous females $(P=0.0028)$ and a statistically significant difference between genotypes at $96 \mathrm{~h}(P=0.0003)$, when heterozygotes produced $\mathrm{JH}$ at a rate $33 \%$ greater than Dahomey. The rates of $\mathrm{JH}$ synthesis were lower in this experiment, and in that reported in figure 1C, because a different batch of radiolabeled methionine was used (fig. $1 \mathrm{~B}$ and C). In a third experiment (fig. 1C), JH biosynthesis by CA from $24 \mathrm{~h}$ chico ${ }^{1}$ homozygotes and Dahomey was compared. The positive control third larval instar brain/ring gland complexes also produced substantially less $\mathrm{JH}$ when the second lot of radiolabel was used (data not shown). There was no significant difference between genotypes when the raw data were analyzed ( $t$-test for unequal variances, $P=0.1618$; Wilcoxon two-sample, $P=0.6269$ ). Normalization of the data to body weight again showed that mutant females produce $\mathrm{JH}$ at a greater rate on a per weight basis ( $t$-test for unequal variances, $P=0.0266$; Wilcoxon two-sample test, $P=0.0422$ ). 

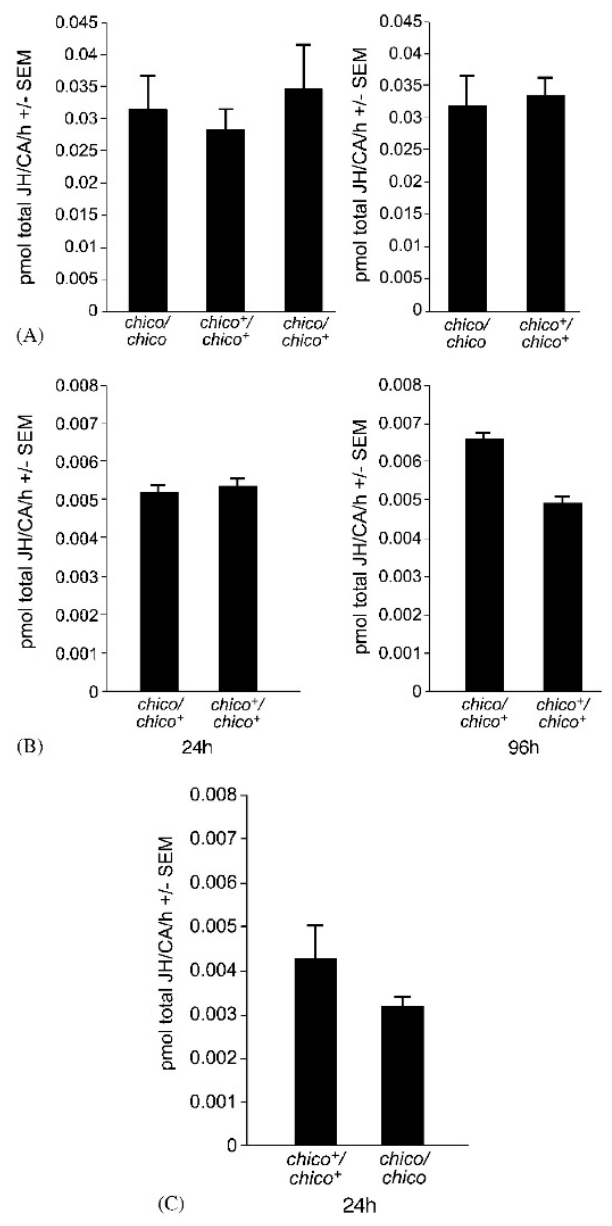

Figure 1. JH production by corpora allata isolated from adult female chico homozygotes, chico/+ heterozygotes and Dahomey, measured by radiochemical assay. (A) chico/chico at $24(n=12)$ and $96 \mathrm{~h}(n=12)$, chico/+ at $24 \mathrm{~h}(n=13),+/+$ at $24(n=12)$ and $96 \mathrm{~h}(n=16)$ post eclosion. (B) chico/+ at $24(n=11)$ and $96 \mathrm{~h}(n=13),+/+$ at $24(n=14)$ and $96 \mathrm{~h}(n=14)$ post eclosion. (C) chico/chico $(n=9)$ and $+/+(n=11)$ at 24 h post eclosion. In each experiment, incubation was for $2 \mathrm{~h}$ at $25^{\circ} \mathrm{C}$. Data shown in A were obtained using one batch of tritiated methionine, whereas data in B and C were obtained using a second batch of label.

\subsection{Ecdysteroids}

Ecdysteroids could be responsible for the control of YP uptake (Richard et al., 1998) and a deficiency could cause the sterility phenotype of chico. The amount of ecdysteroids released from in vitro incubated ovaries removed from the mutant homozygotes, the heterozygote (chico/Dahomey), and wildtype Dahomey were determined at 24 and $96 \mathrm{~h}$ (fig. $2 \mathrm{~A})$. There were statistically significant differences in the amount of ecdysteroids released by the three genotypes $(P=0.0021)$. chico ${ }^{1}$ ovaries released less ecdysteroids than the heterozygote ovaries $(P=0.0068)$ and Dahomey $(P=0.0032)$, especially at $24 \mathrm{~h}$ post-eclosion 
(fig. 2A). When normalized by body weight, there was no overall difference among genotypes $(P=0.2849)$, but there was an interaction between day and genotype $(P=0.0501)$ reflecting relatively low release of ecdysteroids on day 1 by chico ${ }^{1}$ females and relatively high levels compared to the other genotypes on day 4 (fig. 2A).

The abundance of haemolymph ecdysteroids was measured for all three genotypes because the circulating level of systemic hormones is more relevant to physiological activity than the rate of biosynthesis or release (fig. 2B). There was an overall effect of age ( $P=$ $0.0124)$, but not of genotype $(P=0.4947)$. There was no statistically significant genotypeby-age interaction although the ecdysteroids present in day 1 in chico $^{1}$ females were low compared to those in the other genotypes. The effect of age may be due to the greater amount of haemolymph that can be removed from immature versus mature adult females (Handler, 1982). When haemolymph ecdysteroid abundance was normalized by size (fig. 3B), there was an indication of statistically significant differences between genotypes ( $P=$ 0.0568 ) due largely to the relatively high level in chico $^{1}$ females, compared to the other genotypes, at day 4 post-eclosion (fig. 2B).
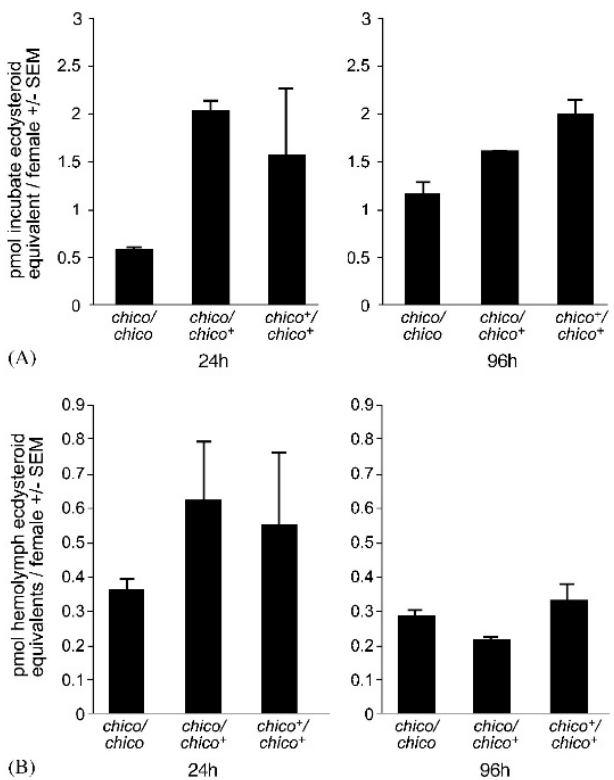

Figure 2. (A) Ecdysteroids released by isolated ovaries: chico/chico $(n=2)$, chico/+ $(n=2)$, $+/+(n=2)$ at 24-post eclosion; $(n=7)$, chico/+ $(n=2)$, and $+/+(n=16)$ at $96 \mathrm{~h}$ post eclosion where $\mathrm{n}$ is the number of replicate determinations. (B) Haemolymph ecdysteroid titers: chico/chico, chico/+ and $+/+$ at $24 \mathrm{~h}$ post eclosion ( $n=2$ per genotype); chico/chico, chico/+, and $+/+$ at $96 \mathrm{~h}$ post eclosion ( $n=3$ per genotype). Haemolymph was drawn from 150 to 200 females of each genotype for each replicate determination $(n)$. 

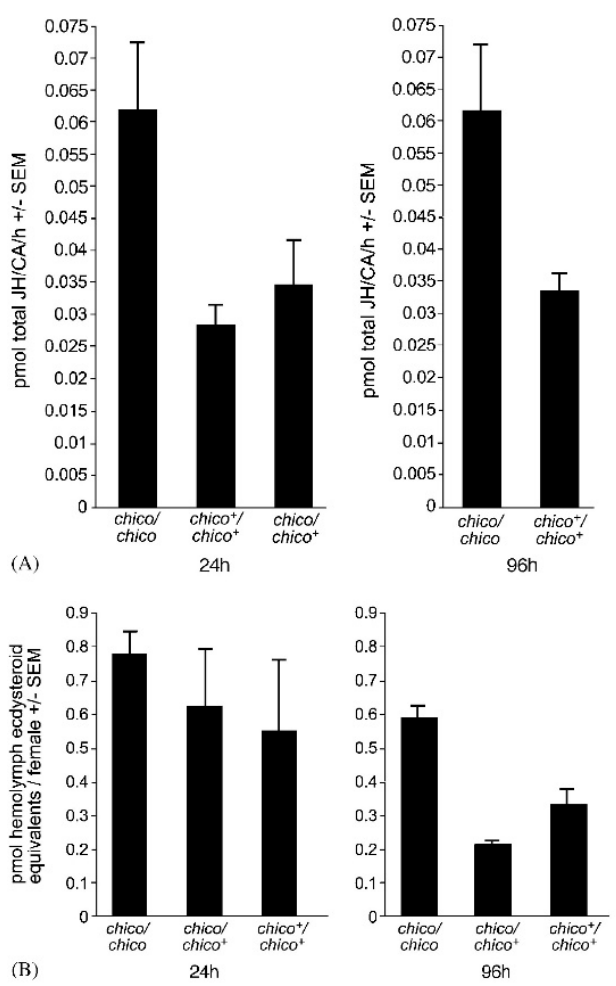

Figure 3. (A) chico/chico females are half the size of the heterozygote and wild-type females. JH biosynthesis data (fig. 1A) normalized by body size. (B) Hemolymph ecdysteroid data (fig. 2B) normalized by body size.

\subsection{Ovariole and nurse cell numbers}

Ecdysteroids are reported to be synthesized by nurse cells (Freeman et al., 1999) and thus we determined the number of nurse cells as a possible explanation for low ecdysteroids on day 1 post-eclosion. The number of ovarioles and number of nurse cells in stage seven egg chambers were counted to characterize the rate and state of ovarian maturation of homozygous chico ${ }^{1}$ females (table 2). On day 1 post-eclosion, chico ${ }^{1}$ females had an average of 11.2 nurse cells per egg chamber and on day 4 they had 14.8 nurse cells per egg chamber, a statistically significant difference $(t=5.8277 ; P<0.025)$. The heterozygote and Dahomey had 15 nurse cells per egg chamber. The low number of nurse cells observed on day 1 in the present study corroborates an earlier observation that DInr mutations exhibit delayed egg chamber maturation (Chen et al., 1996). By day 4, posteclosion chico ${ }^{1}$ egg chambers had approximately the standard number (15) of nurse cells. When the mean number of ovarioles per ovary was compared for chico ${ }^{1}$, heterozygote and wild-type genotypes (table 2), the statistical analysis revealed an interaction between genotype and age $(P<0.0001)$ partially resulting from a relatively low number of ovarioles in $c h i c o^{1}$ females on day 4 . 


\begin{tabular}{|c|c|c|}
\hline \multicolumn{3}{|c|}{$\begin{array}{l}\text { (1) Mean (SEM) nurse cell number per egg chamber in stage } 7 \text { oocytes in chico }{ }^{1} \text { homozygotes } \\
\text { (the heterozygote and Dahomey had } 15 \text { nurse cells per egg chamber) }\end{array}$} \\
\hline & Day 1 & Day 4 \\
\hline chico $^{1} /$ chico $^{1}$ & $11.2(0.54)$ & $14.8(0.30)$ \\
\hline \multicolumn{3}{|c|}{ (2) Mean (SEM) number of ovarioles per ovary } \\
\hline Genotype & Day 1 & Day 4 \\
\hline chico $^{1 / c_{\text {chico }}}$ & $17.2(0.78)$ & $14.7(0.44)$ \\
\hline chico $^{1} /$ Dahomey & $20.0(0.45)$ & $19.9(0.42)$ \\
\hline Dahomey & $19.7(0.43)$ & $19.7(0.38)$ \\
\hline
\end{tabular}

*SEM, standard error of the mean

\subsection{Yolk protein and yolk protein receptor $m R N a$}

An absence of YP or yolk protein receptor could have been responsible for the sterility phenotype of chico. The level of YP (47 kDa) in the ovaries of $c h i c 0^{1}$ females was lower than in the other genotypes (fig. 4A). YP-receptor mRNA was present at low levels in homozygous $c h i c 0^{1}$ ovaries compared to heterozygote and wild-type Dahomey ovaries as indicated by the relative band intensity of the amplicons based on normalization to $1 \mu \mathrm{g}$ polyA mRNA in each amplification (fig. 4B). 


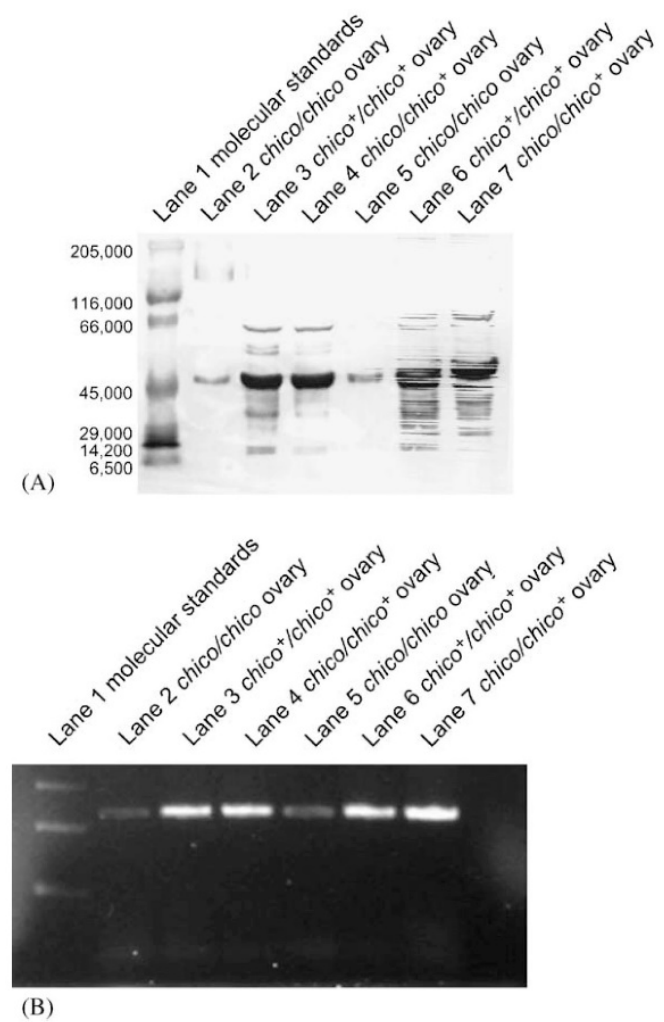

Figure 4. (A) Western blot of YP in chico/chico, chico/+, and +/+ ovaries at 24 and $96 \mathrm{~h}$ posteclosion. The YP band, prominent (1 $\mu \mathrm{g}$ total protein per lane for all genotypes) in the reproductively capable heterozygotes and wild-type ovaries, was observed at $47 \mathrm{kDa}$. (B) YP receptor gene (yolkless) mRNA abundance in chico/chico, chico/+, and +/+ ovaries at 24 and $96 \mathrm{~h}$ post-eclosion. Amplicons from the yolkless gene (shown on the gel) were derived from reverse transcriptase PCR using $1 \mu \mathrm{g}$ mRNA per amplification from each genotype.

\section{Discussion}

The ovary transplant experiments indicate that $\mathrm{CHICO}$ in the ovary is necessary for vitellogenic maturation. From these experiments we conclude that IIS (functional CHICO) is necessary for vitellogenesis even when sufficient levels of $\mathrm{JH}$, ecdysteroids, or other factors are present.

\subsection{Sterility of chico ${ }^{1}$ females was autonomous to the ovary}

Ovaries from immature, reproductively capable females became vitellogenic after transplantation into $c h i c 0^{1}$ females (table 1). The endogenous milieu in $c h i c o^{1}$ females was therefore capable of supporting the vitellogenic maturation of normal ovaries. In contrast, ovaries transplanted from chico $^{1}$ homozygous females into reproductively capable females failed to mature to the first vitellogenic stage. The effect of the chico ${ }^{1}$ mutation on vitellogenesis in homozygous females is therefore endogenous to the ovary and the sterility of 
chico $^{1}$ females is attributable to the absence of CHICO in the ovary itself. IIS may therefore play a role in regulation of YP uptake into oocytes.

Lowered levels of JH biosynthesis in vitro by DInr heteroallelic mutant females compared to wild-type females has been reported (Tatar et al., 2001). These authors argued that lowered JH production in the DInr mutant females, approximately $23 \%$ of wild type, was responsible for their sterility. This view was supported by the finding that addition of the $\mathrm{JH}$ analog methoprene, stimulated a low level of egg production. The results of the present study demonstrate that the sterility of $c h i c{ }^{1}$ females is autonomous to the ovary itself. Our results therefore do not support the argument that IIS in D. melanogaster nonautonomously affects reproduction as inferred from work on insulin receptor mutations (Tatar et al., 2001). However, the insulin receptor mutation is not the same as the insulin receptor mutation used in the present study, and there could be different effects associated with each. Nevertheless, the effect of the $c h i c o^{1}$ mutation on female reproduction was entirely ovaryautonomous.

\subsection{JH biosynthesis or circulating ecdysteroids in mature chico ${ }^{1}$ females was approxi- mately at wild-type levels}

The absolute rate of $\mathrm{JH}$ biosynthesis in chico $^{1}$ females was similar to that in other genotypes (fig. 1). When normalized for body size, the dwarf chico ${ }^{1}$ females were actually found to produce more JH per body mass than the other genotypes (fig. 3A). Our data therefore do not support the finding of Tatar et al. (2001) of lowered JH biosynthesis in females mutant for IIS and hence provide no evidence that lowered production of JH plays any role in the sterility of chico $^{1}$ females. In any case, $\mathrm{JH}$ biosynthesis rate may not reflect the circulating titer of this hormone since at least two JH-degrading enzymes exist in D. melanogaster. The circulating level of hormones is a more meaningful physiological measure, but to date there have been no measures of JH titers in D. melanogaster haemolymph. A stronger indication that JH signaling plays no role in the sterility of $\mathrm{chico}^{1}$ females came from the results of application of the JH agonist methoprene, which did not rescue sterility or vitellogenic egg production in the present study. In general, the present study provides no evidence for a systemic JH effect on reproduction as described earlier (Tatar et al., 2001). Furthermore, chico ${ }^{1}$ females were found to be longer-lived than wildtype controls (Clancy et al., 2001), but the mutant does not produce $\mathrm{JH}$ at a significantly lower rate than wild-type females, thus suggesting that JH biosynthesis does not play a role in the extension of lifespan by $c h i c 0^{1}$ (the longevity phenotype was observed under similar conditions to those used in the present study).

The amount of circulating ecdysteroid did differ significantly between chico $^{1}$ females and the other genotypes on day 4 (fig. 2B). If the amount of haemolymph was normalized by body size, then again, chico ${ }^{1}$ females had more ecdysteroids in the haemolymph (fig. 3B). Our results therefore provide no support for the suggestion that defective IIS results in a diapause-like state characterized by low levels of JH biosynthesis and ecdysteroid abundance (Tatar et al., 2001). Furthermore, the levels of JH and ecdysteroids in chico $\mathrm{fe}^{\mathrm{fe}}$ males were sufficient to support vitellogenesis of ovaries transplanted into homozygous mutant females. 
The transition from the previtellogenic to the vitellogenic state of oocytes occurs either as a developmental event in young adult females, or as a reversal of reproductive diapause (Saunders et al., 1989). Diapause in D. melanogaster is associated with decreased in vitro ovarian ecdysteroid and corpora allata JH production (Saunders et al., 1990) and application of ecdysteroids to diapausing females, or temperature elevation, stimulates vitellogenesis and results in the appearance of the protein components of YP endocytosis (Richard et al., 1998, 2001a, b). However, incubation of diapausing ovaries in the presence of 20-hydroxyecdsone does not initiate vitellogenesis implying that something else may be required (Richard et al., 2001b). We suggest that IIS may play this role.

\subsection{The homozygous mutant ovary was structurally similar to wild type}

The reproductive system of $c h i c 0^{1}$ homozygotes was normal in terms of the numbers of ovarioles and nurse cells per egg chamber on day 4 post-eclosion (table 2). An unusually low number of nurse cells were present in $c h i c 0^{1}$ on day 1 perhaps reflecting the slow development typical of this mutation (Bohni et al., 1999). However, the number of nurse cells was approximately normal in chico $^{1}$ ovaries by day 4 suggesting that in this regard the ovaries attained normal maturation. A lag in nurse cell maturation could have contributed to low levels of ecdysteroid released by the $\mathrm{chico}^{1}$ homozygote ovary on day one (fig. 2A) as nurse cells are a possible site of ecdysteroid biosynthesis (Freeman et al., 1999). The number of ovarioles in $c$ hico ${ }^{1}$ was within the normal range of 15-20 per ovary (Bate and Arias, 1993) as were the numbers for the heterozygote and wild-type Dahomey (table 2). If the ovariole number reflects the number of units in an ovariole that can synthesize hormone, then there was no evidence that the amount of ecdysteroids in the haemolymph of chico ${ }^{1}$ homozygotes was a result of, or even compensation by, an unusually high number of ovarioles. The relatively low level of ovariole numbers in $c h i c 0^{1}$ females on day 4 posteclosion, compared to day 1 in the homozygous mutants, is surprisingly given that ovariole number is determined before eclosion and the relevant adult tissue is non-mitotic.

D. melanogaster is a model system for investigation of the control of vitellogenesis by hormones and other factors. The hormonal control of vitellogenesis in D. melanogaster has been associated with $\mathrm{JH}, 20$-hydroxyecdysone, the ecdysone receptor, the ecdysone early response genes and the sex peptide (Freeman et al., 1999; Bownes et al., 1996; Soller et al., 1997; Buszczak et al., 1999; Carney and Bender, 2000). JH has been implicated in YP synthesis by the ovary and YP uptake into the oocyte (Postlethwait et al., 1980), and 20- hydroxyecdysone in fat body and ovarian follicle cell YP synthesis (Bownes, 1989). JH can also stimulate ecdysteroid production (Kelly, 1994; Richard et al., 1998). JH has been thought to play a predominant role in controlling vitellogenesis (Kelly, 1994; Nijout, 1994), but its direct effect on YP endocytosis remains uncertain (Raikhel and Dhadialla, 1992). Richard et al. (1998, 2001a, b) hypothesized that ecdysteroids and not JHs may play the major role in controlling vitellogenesis; this is based on observations that both apterous ${ }^{56 f}$ mutant females (Altaratz et al., 1991) and females recovering from diapause (Richard et al., 1998) are JH-deficient yet produce ovarian ecdysteroids and are fertile. Based on the arrest of ovarian maturation in IIS mutations prior to the onset of YP uptake by oocytes, previous studies have suggested that the IIS pathway may play a role in vitellogenesis (Chen et al., 
1996), perhaps in conjunction with control of ecdysteroid biosynthesis (Drummond-Barbosa and Spradling, 2001). Alternatively, insulin could control YP uptake by oocytes in $D$. melanogaster via receptor-mediated endocytosis in a manner analogous to that in vertebrates (Schonbaumet al., 2000). From the transplant experiments, we surmised that levels of $\mathrm{JH}$ and ecdysteroids, and any other requisite factors, were sufficient in chico $^{1}$ homozygotes for vitellogenesis. The transplant experiments demonstrated that IIS was necessary for the transition from the final previtellogenic stage to stage 8 vitellogenic oocytes.

Two non-exclusive hypotheses could explain the failure of YP uptake in chico ${ }^{1}$ homozygotes: [1] defective IIS results in relatively low levels of YP and YP-receptor that constrains YP uptake, and/or [2] defective IIS affects the dynamics of assembly of the protein components of receptor mediated endocytosis at the cell surface. In wild-type flies, YP-receptor mRNA and the receptor itself are synthesized at previtellogenic stages considerably before YP uptake (Schonbaum et al., 2000). This indicates that previtellogenic stages do not fail to take up yolk because of an absence of the receptor and supports the idea that YP-receptor relocalization from within the oocyte to the cortex marks the transition to vitellogenesis (Schonbaum et al., 2000). IIS can play a role in regulation of membrane trafficking including recycling of proteins occurring in a number of mammalian transmembrane receptor complexes such as the InR (Corvera and Czech, 1998). A similar mechanism may underlie Drosophila (insect) YP endocytosis.

\section{Longevity}

IIS evidently plays an evolutionarily conserved role in the determination of fecundity and lifespan and it is pertinent to discuss both phenotypes in the context of our study. The effect of reduced IIS signaling was first discovered to extend lifespan in the nematode worm Caenorhabditis elegans (Klass and Hirsh, 1976; Friedman and Johnson, 1988; Kenyon et al., 1993; Kimura et al., 1997), and reduced IIS has since been demonstrated to increase longevity and to induce female sterility in Drosophila (Clancy et al., 2001; Tatar et al., 2001). In the mouse, heterozygosity for the insulin-like growth factor 1 receptor (Holzenberger et al., 2003) and ablation of the insulin receptor in white adipose tissue (Bluher et al., 2003) both extend lifespan. In the mouse, mutations in insulin/IGF signaling can also cause female sterility (Brüning et al., 2000; Burks et al., 2000). The pathway evidently plays an evolutionarily conserved role in the determination of fecundity and lifespan.

The pathways and mechanisms through which lowered IIS can extend lifespan and reduce fecundity remain to be elucidated. In C. elegans, extension of lifespan by reduced IIS is mediated, at least in part, by dephosphorylation and relocation to the nucleus of a winged fork head transcription factor daf-16 (Lin et al., 1997; Ogg et al., 1997). The fork head transcription factor daf-16 transduces insulin-like metabolic and longevity signals in C. elegans. Expression of daf-16 in neuronal tissue appears to be crucial for the extension of lifespan (Wolkow et al., 2000). Although homologues of this gene are present in both Drosophila and the mouse, their role in extension of lifespan by reduced IIS in these organisms has not yet been determined. In C. elegans, modulations of lifespan and fecundity by IIS are thought to be mediated by a systemic signaling event downstream of daf-16, possibly involving steroid hormones (Gerisch et al., 2001). 
In Drosophila, mutations in genes encoding IIS components that have so far been shown to increase lifespan are also associated with sterility or reduced fecundity (Bohni et al., 1999; Clancy et al., 2001; Tatar et al., 2001; Drummond-Barbosa and Spradling, 2001). It has been suggested that both the increased longevity and the sterility associated with reduced IIS in Drosophila are mediated by reduced production of the sesqueterpenoid JH from the corpora allata (Tatar et al., 2001; Tatar and Yin, 2001). Both vitellogenesis of eggs, which is absent in long-lived IIS mutant female flies, and normal lifespan have been reported to be restored in females mutant for Inr that are treated with the JH analogue methoprene (Tatar et al., 2001). Methoprene did not restore vitellogenesis in chico $^{1}$ mutant females in the present study.

Flies mutant for the nuclear hormone receptor for ecdysone, the other major systemic hormone in insects, have been recently reported to be long-lived (Simon et al., 2003), and females mutant for this receptor have previously been demonstrated to be sub-fertile or sterile (Carney and Bender, 2000). Production of ecdysone by the ovary, the main site of synthesis, has been reported to be reduced in flies mutant for the IIS receptor (Tu et al., 2002). It remains to be determined if reduced IIS and ecdysone signaling affect fecundity and lifespan by overlapping or separate mechanisms. Hence in Drosophila as well as C. elegans, there is evidence implicating a second systemic signaling event in mediating the effect of reduced IIS on lifespan and fecundity.

In the present study, hemolymph ecdysteroid levels in chico females were also similar to those in wild-type females. We could therefore find no evidence that these two hormones (JH, ecdysteroids) play a role in producing either the sterility or the increased lifespan of chico females. Our results imply either that reduction of IIS modulate fecundity by different mechanisms, both within Drosophila and between Drosophila and C. elegans, or that the systemic environment is in fact irrelevant to the fecundity defects associated with mutant IIS in both organisms.

\section{Conclusions}

Our study indicates that defective insulin/insulin-like growth factor-like signaling (IIS) in chico ${ }^{1}$ mutant females blocks vitellogenesis irrespective of sufficient levels of juvenile hormone and ecdysteroids to support vitellogenic egg maturation. Regardless of whether IIS acts directly on the yolk protein uptake system or through some intermediate, such as the ecdysone receptor, it is clear that IIS plays an ovarian autonomous role in vitellogenesis.

Acknowledgments - Mary Bownes and Alexander Raikhel kindly provided antibody used in this study. Dave Marx, Department of Biometry at the University of Nebraska-Lincoln, kindly conducted statistical analyses. We thank Marianna Wolfner for comments on a draft of the manuscript. L. Harshman was supported by a grant from the National Institute of Health (NIA AG08761) and a NSF Grant (EPS-0346476). D. Richard was supported by NIH Grant GM/OD54905. L. Partridge was supported by the BBSRC and the Wellcome Trust. 


\section{References}

Altaratz, M., Segal, D., Richard, D.S., Gilbert, L.I., Applebaum, S.W., 1991. Regulation of juvenile hormone synthesis in wild-type and apterous mutant Drosophila. Molecular and Cellular Endocrinology 81, 205-216.

Bate, M., Arias, A.M., 1993. The Development of Drosophila melanogaster. Cold Spring Harbor Press, New York.

Bluher, M., Kahn, B.B., Kahn, C.R., 2003. Extended longevity in mice lacking the insulin receptor. Science 299, 572-574.

Bohni, R., Riesgo.-Escovar, J., Oldham, S., Brogiolo, W., Stocker, H., Andruss, B.F., Beckingham, K., Hafen, E., 1999. Autonomous control of cell and organ size by CHICO, a Drosophila homolog of vertebrate IRS1-4. Cell 97, 865-875.

Bownes, M., 1989. The roles of juvenile hormone, ecdysone and the ovary in the control of Drosophila vitellogenesis. Journal of Insect Physiology 35, 409-413.

Bownes, M., Ronaldson, E., Mauchline, D., 1996. 20-Hydroxyecdysone, but not juvenile hormone, regulation of yolk protein gene expression can be mapped to cis-acting DNA sequences. Developmental Biology 173 (2), 475-489.

Brüning, J.C., Gautam, D., Burks, D.J., Gillette, J., Schubert, M., Orban, P.C., Klein, R., Krone, W., Müller-Wieland, D., Kahn, C.R., 2000. Role of insulin receptor in control of body weight and reproduction. Science 289, 2122-2125.

Burks, D.J., de Mora, J.F., Schubert, M., Withers, D.J., Myers, M.G., Towery, H.H., Alramuro, S.L., Flint, C.L., White, M.F., 2000. IRS-2 pathways integrate female reproduction and energy homeostasis. Nature 407, 377-382.

Buszczak, M., Freeman, M.R., Carlson, J.R., Bender, M., Cooley, L., Segraves, W.A., 1999. Ecdysone response genes govern egg chamber development during mid-oogenesis in Drosophila. Development 126, 4581-4589.

Carlson, K.A., Harshman, L.G., 1999. Extended longevity lines of Drosophila melanogaster: characterization of oocyte stages and ovariole numbers as a function of age and diet. Journal of Gerontology A Biological Sciences and Medical Sciences 54 (10), B432-B440.

Carney, G.E., Bender, M., 2000. The ecdysone receptor (EcR) gene is required maternally for normal oogenesis. Genetics 154, 1203-1211.

Chen, C., Jack, J., Garofalo, R.S., 1996. The Drosophila insulin receptor is required for normal growth. Endocrinology 137, 846-856.

Clancy, D.J., Gems, D., Harshman, L.G., Oldham, S., Stocker, H., Hafen, E., Leevers, S.J., Partridge, L., 2001. Extension of lifespan by CHICO, a Drosophila insulin receptor substrate protein. Science 292, 104-106.

Corvera, S., Czech, M.P., 1998. Direct targets of phosphoinositide 3-kinase products in membrane traffic and signal transduction. Trends in Cell Biology 8, 442-446.

Coyne, J.A., Rux, J., David, J.R., 1991. Genetics of morphological differences and hybrid sterility between Drosophila sechellia and its relatives. Genetical Research 57, 113-122.

Drummond-Barbosa, D., Spradling, A.C., 2001. Stem cells and their progeny respond to nutritional changes during Drosophila oogenesis. Developmental Biology 231, 265-278.

Freeman, M.R., Dobritsa, A., Gaines, P., Segraves, W.A., Carlson, J.R., 1999. The dare gene: steroid hormone production, olfactory behavior, and neural degeneration in Drosophila. Development $126,4591-4602$. 
Friedman, D.B., Johnson, T.E., 1988. A mutation in the age-1 gene in Caenorhabditis elegans lengthens life and reduces hermaphrodite fertility. Genetics 118, 75-86.

Gerisch, B., Weitzel, C., Kober-Eisermann, C., Rottiers, V., Antebi, A., 2001. A hormonal signaling pathway influencing C. elegans metabolism, reproductive development, and life span. Development of Cell 1, 841-851.

Handler, A.M., 1982. Ecdysteroid titers during pupal and adult development in Drosophila melanogaster. Developmental Biology 93, 73-82.

Harshman, L.G., Loeb, A.M., Johnson, B.A., 1999. Ecdysteroid titers in mated and unmated Drosophila melanogaster females. Journal of Insect Physiology 45, 571-577.

Holzenberger, M., Dupont, J., Ducos, B., Leneuve, P., Geloen, A., Even, P.C., Cervera, P., Le Bouc, Y., 2003. IGF-1 receptor regulates lifespan and resistance to oxidative stress in mice. Nature $421,182-$ 187.

Kelly, T.J., 1994. Endocrinology of Vitellogenesis in Drosophila Melanogaster. Perspectives In Comparative Endocrinology. National Research Council of Canada, pp. 282-290.

Kenyon, C., Chang, J., Gensch, E., Rudner, A., Tabtiang, R., 1993. A C. elegans mutant that lives twice as long as wild type. Nature $366,461-464$.

Kimura, K.D., Tissenbaum, H.A., Liu, Y., Ruvkun, G., 1997. daf-2, an insulin receptor-like gene that regulates longevity and diapause in Caenorhabditis elegans. Science 277, 942-946.

King, R.C., 1970. Ovarian Development in Drosophila melanogaster. Academic Press, New York.

Kiriishi, S., Rountree, D.B., Sakurai, S., Gilbert, L.I., 1990. Prothoracic gland synthesis of 3-dyhydroecdysone and its haemolymph 3 beta-reductase mediated conversion to ecdysone in representative insects. Experientia 46, 716-721.

Klass, M.R., Hirsh, D.I., 1976. Non-aging developmental variant of C. elegans. Nature 260, 523-525.

Lin, K., Dorman, J.B., Rodan, A., Kenyon, C., 1997. daf-16: an HNF-3/forkhead family member that can function to double the life-span of Caenorhabditis elegans. Science 278, 1319-1322.

Nijout, J.F., 1994. Insect Hormones. Princeton University Press, Princeton, New Jersey.

Ogg, S., Paradis, S., Gottlieb, S., Patterson, G.I., Lee, L., Tissenbaum, H.A., Ruvkun, G., 1997. The fork head transcription factor DAF-16 transduces insulin-like metabolic and longevity signals in C. elegans. Nature 389, 994-999.

Postlethwait, J.H., Bownes, M., Jowett, T., 1980. Sexual phenotype and vitellogenin Synthesis in Drosophila melanogaster. Developmental Biology 79, 379-387.

Raikhel, A.S., Dhadialla, T.S., 1992. Accumulation of yolk proteins in insect oocytes. Annual Review of Entomology 37, 217-251.

Richard, D.S., Applebaum, S.W., Slither, T.J., Baker, F.C., Schooley, D.A., Reuter, C.C., Henrich, V.C., Gilbert, L.I., 1989. Juvenile hormone bisexpoxide biosynthesis in vitro by the ring gland of Drosophila melanogaster: a putative juvenile hormone in the higher Diptera. Proceedings of the National Academy of Sciences USA 86, 1421-1425.

Richard, D.S., Watkins, N.L., Serafin, R.B., Gilbert, L.I., 1998. Ecdysteroids regulate yolk protein uptake by Drosophila melanogaster oocytes. Journal of Insect Physiology 44, 637-644.

Richard, D.S., Gilbert, M., Crum, B., Hollinshead, D.M., Schelble, S., Scheswohl, D., 2001a. Yolk protein endocytosis by oocytes in Drosophila melanogaster: immunofluorescent localization of clathrin, adaptin and the yolk protein receptor. Journal of Insect Physiology 47, 715-723.

Richard, D.S., Jones, J.M., Barbarito, M.R., Cerula, S., Detweiler, J.P., Fisher, S.J., Brannigan, D.M., Scheswohl, D.M., 2001b. Vitellogenesis in diapausing and mutant Drosophila melanogaster: further 
evidence for the relative roles of ecysteroids and juvenile hormones. Journal of Insect Physiology 47, 905-913.

Saunders, D.S., Henrich, V.C., Gilbert, L.I., 1989. Induction of diapause in Drosophila melanogaster: photoperiodic regulation and the impact of arrhythmic clock mutations on time measurement. Proceedings of the National Academy of Sciences USA 86, 3748-3752.

Saunders, D.S., Richard, D.S., Applebaum, S.W., Ma, M., Gilbert, L.I., 1990. Photoperiodic diapause in Drosophila melanogaster involves a block to the juvenile hormone regulation of ovarian maturation. General and Comparative Endocrinology 79, 174-184.

Schonbaum, C.P., Perrino, J.J., Mahowald, A.P., 2000. Regulation of the vitellogenin receptor during Drosophila melanogaster oogenesis. Molecular Biology of the Cell 11, 511-521.

Simon, A.F., Shih, C., Mack, A., Benzer, S., 2003. Steroid control of longevity in Drosophila melanogaster. Science 299, 1407-1410.

Soller, M., Bownes, M., Kubli, E., 1997. Mating and sex peptide stimulate the accumulation of yolk in oocytes of Drosophila melanogaster. European Journal of Biochemistry 243, 732-738.

Tatar, M., Yin, C., 2001. Slow aging during insect reproductive diapause: why butterflies, Grasshoppers and flies are like worms. Experimental Gerontology 36, 723-738.

Tatar, M., Kopelman, A., Epstein, D., Tu, M.-P., Yin, C.-M., Garofalo, R.S., 2001. A mutant Drosophila insulin receptor homolog that extends life-span and impairs neuroendocrine function. Science 292, 107-110.

Tobe, S.S., Pratt, G.E., 1974. The influence of substrate concentrations on the rate of insect juvenile hormone biosynthesis by the corpora allata of the desert locus in vitro. Biochemical Journal 144, 107-133.

Tu, M.-P., Yin, C.-M., Tatar, M., 2002. Impaired ovarian ecdysone synthesis of Drosophila melanogaster insulin receptor mutants. Aging Cell 1, 158-160.

Warren, J.T., Smith, W.A., Gilbert, L.I., 1984. Simplification of the ecdysteroid radioimmunoassay by the use of protein A from Staphylococcus aureus. Experientia 40, 393-394.

Wilson, T.G., 1982. A correlation between juvenile hormone deficiency and vitellogenic Oocyte degeneration in Drosophila melanogaster. Wilhelm Roux's Archives of Developmental Biology 191, 257-263.

Wilson, T.G., Chaykin, D., 1985. Toxicity of methoprene to Drosophila melanogaster (Diptera: Drosophiliidae): a function of larval culture density. Journal of Economic Entomology 78, 1208-1211.

Wilson, T.G., Fabian, J., 1986. A Drosophila melanogaster mutant resistant to a chemical analog of juvenile hormone. Developmental Biology 118, 190-201.

Wolkow, C.A., Kimura, K.D., Lee, M.S., Ruvkum, G., 2000. Regulation of C. elegans life-span by insulin signaling in the nervous system. Science 290, 147-150. 